\title{
Induction of Myocardial PDCD4 in Coronary Microembolization-Related Cardiac Dysfunction: Evidence from a Large-Animal Study
}

\author{
Qiang Su Lang Li You Zhou Jiangyou Wang Yangchun Liu Guotian Ma \\ Department of Cardiology, the First Affiliated Hospital of Guangxi Medical University, Nanning, China
}

\section{Key Words}

Coronary microembolization • Percutaneous intervention • Cardiac function • PDCD4

\begin{abstract}
Background/Aims: Coronary microembolization (CME) has been linked to myocardial inflammation and apoptosis. This study aims to investigate the role of the apoptotic protein PDCD4 in the myocardium after CME in minipigs. Methods: Seventy Bama minipigs were randomized into four groups: control, CME, CME plus PDCD4-siRNA and CME plus control siRNA. CME was induced by injecting polyethylene microspheres into the left anterior descending artery. Cardiac function was evaluated. HE and HBFP staining were used to observe the degree of infarction. Western blotting and qPCR were used to evaluate the expression of PDCD4, TNF- $\alpha$ and caspase-3. The measurements were performed at $0,3,6,9,12$ and $24 \mathrm{~h}$ after CME modeling in the CME and control groups. Results: Cardiac function in the CME group was significantly decreased compared with the control group $(P<0.05)$ and the expression of PDCD4 and TNF- $\alpha$ increased significantly $(P<0.05)$. However, the infarct area did not differ between the CME and control groups at any time point $(P>0.05)$. Furthermore, PDCD4-siRNA improved cardiac function and reduced PDCD4 and TNF- $\alpha$ expression compared with the CME plus control siRNA group at $9 \mathrm{~h}$ after modeling $(P<0.05)$, while the caspase- 3 level was not different between the two groups. Conclusion: PDCD4 induction may be involved in CME-related cardiac dysfunction, and PDCD4 inhibition via siRNA may attenuate the cardiac impairment and be used as a treatment strategy for CME.
\end{abstract}




\section{Introduction}

Coronary microembolization (CME) is a frequent clinical event that can be caused by either spontaneous or procedure-related atherosclerotic plaque rupture in patients with acute coronary syndromes, particularly during percutaneous coronary intervention (PCI) [1-4]. The incidence is reported to be up to $45 \%$ in patients with great saphenous vein graft percutaneous intervention. The transient "no blood flow" or "slow flow" caused by the CME is an independent predictor of long-term poor prognosis in patients with acute myocardial infarction [5]. Furthermore, CME can also lead to progressive myocardial malfunction, including overt myocardial infarction, contractile dysfunction, arrhythmias and coronary reserve reduction [6]. Once CME occurs, the clinical outcomes of such patients are hardly improved the by administration of coronary thrombolytic agents, nitroglycerin, platelet GP IIb/IIIa receptor antagonists or direct thrombectomy [6]. Therefore, elucidating the pathophysiologic molecular mechanisms underlying CME is significant for effective therapy for $\mathrm{CME}$, and, more importantly, for the improvement of patient outcomes.

Experimental CME induced by polystyrene microsphere injection could result in progressive myocardial dysfunction associated with inflammation [7, 8]. An animal model of CME that is induced by polystyrene microspheres has been widely used to explore the mechanism of CME. Inflammatory responses, characterized by increased leukocyte infiltration and increased levels of cytokines, may play key roles in the pathogenesis of CME-related cardiac dysfunction [8]. Indeed, tumor necrosis factor- $\alpha$ (TNF- $\alpha$ ) is increased after CME and is causally involved in progressive contractile dysfunction [7]. Pretreatment with a TNF- $\alpha$ antibody can prevent contractile dysfunction after microembolization, and the intracoronary infusion of exogenous TNF- $\alpha$ induces contractile dysfunction in the absence of microembolization [8]. Canton et al. found that myofibrillar protein oxidation may represent a mechanistic link between inflammation and contractile dysfunction following CME [9]. Thielmann et al. reported that a signal transduction cascade of NO, TNF- $\alpha$, and sphingosine is causally involved in the CME-induced progressive contractile dysfunction [10]. However, the role of PDCD4 in myocardial dysfunction in the CME is unknown. PDCD4 is a critical mediator of cancer cell apoptosis [11]. Interestingly, the role of PDCD4 in cardiac dysfunction has also been described in recently studies. As a target gene of miR-21, the cardiac-protective role of PDCD4 was verified in cultured cardiac cells and a cardiac I/R model [12]. Moreover, intensive atorvastatin treatment reduces the post-PCI myocardial inflammatory response in patients with unstable angina, which may relate to the inhibition of PDCD4 expression in CD4 ${ }^{+}$T lymphocytes $[13,14]$.

Therefore, this study aims to characterize the expression of PDCD4 in the myocardium after CME in minipigs as well as to explore the dynamic association between PDCD4 expression and cardiac function, which may provide a theoretical basis for the prevention and treatment of CME based on targeting PDCD4.

\section{Materials and Methods}

\section{Animals}

Adult female or male Bama minipigs (weighing 25-30 kg, Animal Center, Guangxi University) were used in the study. All experimental protocols were approved by the Institutional Animal Care and Use Committee at the Medical University of Guangxi, and were consistent with the Guide for the Care and Use of Laboratory Animals (National Institutes of Health publication 85-23, revised 1985). Seventy Bama minipigs were randomized into four groups: control $(n=30)$, CME $(n=30)$, CME plus PDCD4-siRNA ( $=5)$ and CME plus control siRNA $(n=5)$ groups. For minipigs in the CME and control groups, 5 animals were randomly selected for subsequent measurements and analyses at each time point $(0,3,6,9,12$, and $24 \mathrm{~h}$ after the procedure). For minipigs in the CME plus PDCD4-siRNA and CME plus control siRNA groups, subsequent measurements were performed at $9 \mathrm{~h}$ after modeling. 
Su et al.: PDCD4 Expression in Coronary Microembolization

\section{Coronary microembolization induction}

Catheter sheaths (6-F) were implanted and fixed in either side of the femoral artery. All pigs were fully heparinized after the implantation of the sheaths during the full duration of the experiments. After intubation of the left coronary artery with an XB-3 coronary catheter (Cordis Neurovascular, Miami, Florida), insertion of a coronary wire (Floppy Wire, Guidant, Santa Clara, California) and advancement of a 2.3-F infusion microcatheter (Prowler Plus, Cordis Neurovascular), CME was induced by injection of microspheres $(42-\mu \mathrm{m}$ diameter, Biosphere Medical Inc) to the left anterior descending (LAD) artery, as previously described [8]. A total of 100000 microspheres, approximately 4500 microspheres per $\mathrm{ml} / \mathrm{min}$ estimated coronary flow, were injected into each pig, while the same volume of saline was injected into the minipigs in the control group. Infusion of microspheres was followed by a 5-mL heparinized saline flush.

\section{In vivo PDCD4 siRNA delivery}

Seventy-two hours before CME modeling, 10 minipigs were treated with in vivo siRNA: 5 with PDCD4 siRNA and the other 5 with control siRNA. In vivo transfer of siRNA was performed according to methods described previously [15]. After endotracheal intubation, anesthesia was maintained by intravenous dripping with diazepam via the ear vein. The right femoral artery was separated, and a $6 \mathrm{~F}$ vascular sheath was placed. Prior to coronary cannulation, animals were treated with $200 \mathrm{U} / \mathrm{kg}$ intravenous injection of heparin $(100 \mathrm{U} / \mathrm{kg} / \mathrm{h})$ to maintain heparinization. A 6-F JL 4.0 guiding catheter was used for coronary angiography. After coronary angiography, a 1.8-F infusion catheter was placed in LAD artery with the tip located between the second and third diagonal branches. One hundred micrograms of PDCD4-siRNA (Genepharma, China) or control-siRNA (Genepharma, China) was diluted with the same volume of in vivo transfection reagent (Entranster ${ }^{\mathrm{TM}}$-in vivo; Engreen, Beijing, China). After being mixed gently by pipetting, the solutions were infused into the LAD within $1 \mathrm{~min}$ before flushing with $2 \mathrm{~mL}$ saline.

\section{Echocardiographic assessment of cardiac function}

Echocardiography was performed using a SEQUOIA Echocardiography System (ACUSON Corporation) with a 12-MHz transducer. The animals were sedated with the inhalation of isoflurane (2\%) and placed at the left lateral decubitus position. Cardiac output (CO), left ventricle end-diastolic diameter (LVEDD), and left ventricle end-systolic diameter (LVESD) were measured perpendicular to the long axis of the ventricle. Fractional shortening (\%) was calculated as (LVEDD-LVESD)/LVEDD*100. Left ventricular ejection fraction (LVEF) (\%) was calculated automatically by the echocardiography system via the following equation: $\mathrm{LVEF}=(\mathrm{LVEDV}-\mathrm{LVESV}) / \mathrm{LVEDV}^{*} 100$, while $\mathrm{LVEDV}=7.0 * \mathrm{LVEDD}^{3} /(2.4+\mathrm{LVEDD})$ and $\mathrm{LVESV}=7.0 * \mathrm{LVESD}^{3} /$ (2.4+LVESD).

\section{Morphological and histological studies}

After the completion of all measurements, the hearts were arrested with $10 \%$ potassium chloride and rapidly excised. After fixation in $4 \%$ formaldehyde, the heart was sliced into 5-mm slices, stained with HE and then photographed. Cross-sectional areas of the ventricular muscle, scar area, and scar thickness were measured. HBFP staining, which is a sensitive method for the early diagnosis of myocardial ischemia, was also performed. For HBFP staining, five microscopic visual fields $(\times 100)$ were randomly sampled from each slice for observation with a DMR+Q550 pathological image pattern, using the Leica Qwin analysis software (Germany). The planar area method was used to measure the infarction zone, which was expressed as the area percentage of bulk-analysis slices and averaging [16].

RNA extraction, reverse transcription, and real-time quantitative PCR

Total RNA from the left ventricular myocardium was isolated using the TRIzol reagent (Invitrogen) according to the manufacturers' instructions. The yield of RNA was quantified using a NanoDrop (GE Healthcare) and subjected to reverse transcription using a reverse transcription kit (Fermentas). Then, cDNA was subjected to qPCR for PDCD4 and TNF- $\alpha$ mRNA using a SYBR Green PCR kit (Promega). Quantification was performed by normalization against GAPDH. The following primer sequences were used in this study: PDCD4 forward, 5'-GCTACGGTGCTCCTGAGTAT-3'; PDCD4 reverse, 5'-GGCAATGTTCAGCTTCCGAT-3'; TNF- $\alpha$ forward, 5'-GGCCCAAGGACTCAGATCAT-3'; TNF- $\alpha$ reverse, 5'-GCATACCCACTCTGCCATTG-3'; GAPDH forward, 5'- GCAAAGTGGACATTGTCGCCATCA; GAPDH reverse, 5'- TCCTGGAAGATGGTGATGGCCTTT. 
Table 1. Changes in cardiac function after CME modeling in control and CME groups. CME, coronary microembolization; LVEF, left ventricular ejection fraction; $\mathrm{CO}$, cardiac output; LVEDD, left ventricular enddiastolic diameter; LVFS, left ventricular fractional shortening. The superscript ${ }^{a}$ indicates a $\mathrm{p}$ value $<0.05, \mathrm{n}=5$, compared to the control group

\begin{tabular}{|c|c|c|c|c|c|}
\hline & & LVEF (\%) & $\mathrm{CO}(\mathrm{L} / \mathrm{min})$ & LVEDD (mm) & LVFS (\%) \\
\hline \multirow[t]{6}{*}{ Control } & $0 \mathrm{~h}(\mathrm{n}=5)$ & $66.25 \pm 1.83$ & $3.92 \pm 0.92$ & $3.56 \pm 0.29$ & $40.03 \pm 4.59$ \\
\hline & $3 h(n=5)$ & $65.23 \pm 2.47$ & $3.66 \pm 0.78$ & $3.44 \pm 0.38$ & $42.14 \pm 4.48$ \\
\hline & $6 h(n=5)$ & $67.37 \pm 2.50$ & $3.71 \pm 0.89$ & $3.61 \pm 0.33$ & $42.97 \pm 5.07$ \\
\hline & $9 \mathrm{~h}(\mathrm{n}=5)$ & $68.78 \pm 2.35$ & $3.97 \pm 0.79$ & $3.48 \pm 0.31$ & $41.23 \pm 5.76$ \\
\hline & $12 \mathrm{~h}(\mathrm{n}=5)$ & $66.20 \pm 2.74$ & $3.87 \pm 0.96$ & $3.58 \pm 0.27$ & $42.18 \pm 5.74$ \\
\hline & $24 \mathrm{~h}(\mathrm{n}=5)$ & $64.50 \pm 1.96$ & $3.82 \pm 0.70$ & $3.92 \pm 0.41$ & $38.91 \pm 5.63$ \\
\hline \multirow[t]{6}{*}{ CME } & $0 h(n=5)$ & $68.11 \pm 2.02$ & $3.95 \pm 0.88$ & $3.63 \pm 0.34$ & $41.07 \pm 4.74$ \\
\hline & $3 h(n=5)$ & $62.26 \pm 2.30$ & $3.90 \pm 0.69$ & $3.91 \pm 0.23$ & $38.59 \pm 7.06$ \\
\hline & $6 \mathrm{~h}(\mathrm{n}=5)$ & $54.39 \pm 3.99 a$ & $3.41 \pm 0.89 a$ & $4.62 \pm 0.32^{a}$ & $30.26 \pm 8.11^{a}$ \\
\hline & $9 h(n=5)$ & $50.19 \pm 3.37^{a}$ & $2.89 \pm 0.76^{a}$ & $5.05 \pm 0.41^{a}$ & $26.34 \pm 7.92^{\mathrm{a}}$ \\
\hline & $12 \mathrm{~h}(\mathrm{n}=5)$ & $56.41 \pm 3.83^{a}$ & $3.64 \pm 0.73^{a}$ & $4.39 \pm 0.41^{\mathrm{a}}$ & $33.80 \pm 9.71^{a}$ \\
\hline & $24 \mathrm{~h}(\mathrm{n}=5)$ & $61.69 \pm 4.87$ & $3.81 \pm 1.16$ & $3.84 \pm 0.34$ & $37.36 \pm 4.14$ \\
\hline
\end{tabular}

Western blotting

Western blotting was performed as described previously [6]. Briefly, each sample containing $50 \mu \mathrm{g}$ of protein was loaded on $10 \%$ polyacrylamide gels and transferred to a PVDF membrane (Millipore). Membranes were incubated overnight at $4{ }^{\circ} \mathrm{C}$ in $5 \%$ non-fat dry milk containing antibody against PDCD4 (Abcam; $1: 1$ 000, goat anti-swine), TNF- $\alpha$ (LifeSpan BioSciences; $1: 200$, rabbit anti-swine) or caspase-3 (Santa Cruz Biotechnology; $1: 200$, goat anti-swine). Primary antibody was detected with secondary horseradish peroxidase-conjugated antibody (Novus Biologicals). The density of the protein bands was quantified by densitometric analysis, and the results were normalized against GAPDH (Proteintech Group).

\section{Statistical analysis}

Data were analyzed using the SPSS 16.0 statistical package (SPSS, Chicago, Illinois, USA). Continuous variables are presented as mean \pm SEM. Statistical analysis was performed using the $t$ test or one-way analysis of variance (ANOVA) with post-hoc analysis using Tukey's test to analyze statistically significant differences between two or more groups. Two-way ANOVA (group, time point) with a post-hoc tests was used for comparison of cardiac function (Table 1). Differences at $p<0.05$ were considered statistically significant.

\section{Results}

Changes in the cardiac function in each group after CME

The cardiac function of the minipigs in the CME group at $3 \mathrm{~h}, 6$, and $9 \mathrm{~h}$ after CME modeling was significantly decreased compared to the control group at the same time points (Table 1). Myocardial contractile dysfunction was observed in minipigs of the CME groups, as reflected by the reduced LVEF, FS, and CO $(\mathrm{P}<0.05)$ and increased LVEDD compared to the control minipigs $(\mathrm{P}<0.05)$. The values of LVEF, FS, and CO were reduced $(\mathrm{P}<0.05)$ while LVEDD was increased at 12 and $24 \mathrm{~h}$ after the CME modeling in the CME groups, although the differences were not statistically significant. More important, compared with the CME plus control-siRNA group, the minipigs in the CME plus PDCD4-siRNA group exhibited significantly increased LVEF, CO, and FS $(\mathrm{P}<0.05)$, with a decrease in LVEDD at $9 \mathrm{~h}$ after the procedure, suggesting that PDCD4-siRNA somehow improved the cardiac function (Table 2).

\section{Histopathology of post-CME myocardial microinfarction}

In HBFP staining, the ischemic myocardium and erythrocytes were dyed a red color, while the normal myocardial cytoplasm and nuclei were dyed yellow and blue, respectively. The HE and HBFP tissue sample staining patterns from the CME group at $3 \mathrm{~h} 6 \mathrm{~h} 9 \mathrm{~h} 12 \mathrm{~h}$, and $24 \mathrm{~h}$ after the procedure revealed a focal distribution of a wedge-shaped myocardial microinfarction with inflammatory cell infiltration (Fig. 1 and 2). Most of the infarction was found in the left ventricular and endocardial area, and the microsphere was found in the lumen of the micro-arteries. Myocardial edema and degeneration could also be observed in the cells surrounding the infarction area. Some of the cardiomyocyte nuclei were not 
Table 2. Effects of PDCD4-siRNA on cardiac function $9 \mathrm{~h}$ following CME modeling. CME, coronary microembolization; LVEF, left ventricular ejection fraction; CO, cardiac output; LVEDD, left ventricular end-diastolic diameter; LVFS, left ventricular fractional shortening. The superscript ${ }^{a}$ indicates a $\mathrm{p}$ value $<0.05, \mathrm{n}=5$, compared to the CME+ control-siRNA group

\begin{tabular}{lccccc}
\hline & $\mathrm{N}$ & LVEF (\%) & CO (L/min) & LVEDD (mm) & LVFS (\%) \\
\hline CME+control-siRNA & 5 & $49.89 \pm 3.77$ & $2.88 \pm 0.89$ & $5.12 \pm 0.67$ & $26.37 \pm 8.01$ \\
CME+PDCD4-siRNA & 5 & $66.39 \pm 2.67^{\mathrm{a}}$ & $3.82 \pm 0.91^{\mathrm{a}}$ & $3.63 \pm 0.29 \mathrm{a}$ & $42.06 \pm 5.77^{\mathrm{a}}$ \\
\hline
\end{tabular}

Table 3. Cardiac infarction area after coronary microembolization $(\bar{x} \pm \mathrm{s}, \%)$. CME, coronary microembolization

\begin{tabular}{ccccccc}
\hline & $0 \mathrm{~h}$ & $3 \mathrm{~h}$ & $6 \mathrm{~h}$ & $9 \mathrm{~h}$ & $12 \mathrm{~h}$ & $24 \mathrm{~h}$ \\
\hline Control & - & - & - & - & - & - \\
CME & - & $8.59 \pm 3.03$ & $8.82 \pm 2.95$ & $9.23 \pm 3.59$ & $9.07 \pm 3.44$ & $9.16 \pm 3.24$
\end{tabular}

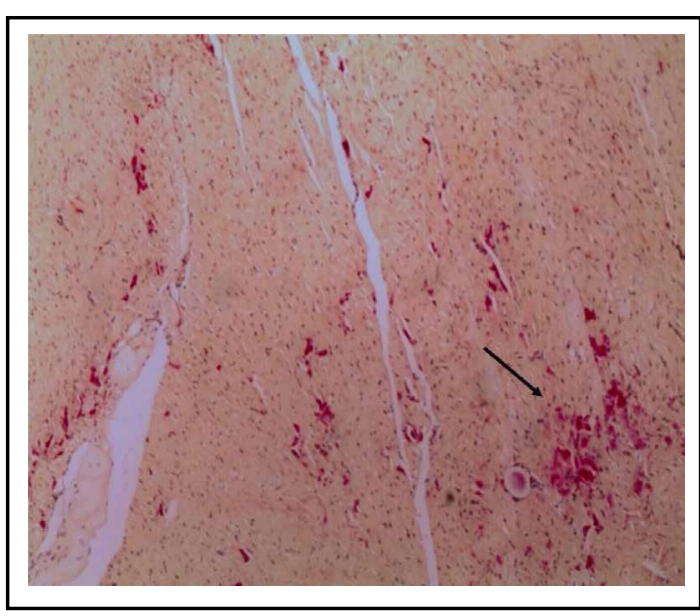

Fig. 1. Histopathologic examination of CME myocardial microinfarction. HBFP staining shows the area of myocardial microinfarction in red. The arrow indicates the presence of a microsphere $(\times 200)$. CME, coronary microembolization.

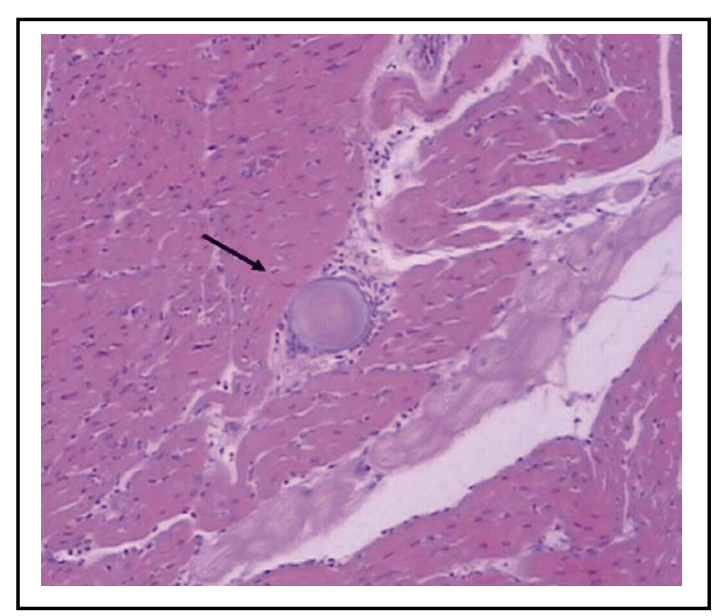

Fig. 2. Histopathologic examination of myocardial microinfarction. HE staining revealed myocardial infarction in the CME group. Microspheres in the samples of myocardium from the CME group are indicated by arrows $(\times 400)$. CME, coronary microembolization.

visible by HE staining. The sizes of the microinfarctions were similar among the myocardium samples at $3 \mathrm{~h} 6 \mathrm{~h} 9 \mathrm{~h} 12 \mathrm{~h}$, and $24 \mathrm{~h}$ in the minipigs in the CME groups (Table 3, P>0.05). No signs of myocardial infarction could be detected in the animals from the control group. Moreover, there was no significant difference in the myocardial infarction size in the minipigs from the CME plus PDCD4-siRNA group compared with those from the CME plus controlsiRNA group $(9.15 \pm 3.27$ vs. $9.73 \pm 3.64, \mathrm{P}>0.05)$.

The myocardial expression of PDCD4 and TNF- $\alpha$ mRNA

qPCR analysis was used to detect myocardial PDCD4 and TNF- $\alpha$ mRNA expression in the minipigs (Fig. 3). Compared with the minipigs from the control group, those from the CME group showed significantly enhanced expression of myocardial PDCD4 and TNF- $\alpha$ mRNA expression at 3, 6, 9, 12 and $24 \mathrm{~h}$ after CME modeling (all $\mathrm{P}<0.05$ ). Compared with $0 \mathrm{~h}$, the myocardial PDCD4 and TNF- $\alpha$ mRNA expression increased at $3 \mathrm{~h}$, peaked at $9 \mathrm{~h}$, and was still increased at 12 and $24 \mathrm{~h}$ after the procedure $(\mathrm{P}<0.05)$. Moreover, PDCD4-siRNA treatment 
Fig. 3. Dynamic changes in PDCD 4 and TNF- $\alpha$ mRNA transcription in the myocardium detected by qPCR in the CME and control groups. (A) PDCD4 and (B) TNF- $\alpha$ normalized against GAPDH. Data are mean \pm SEM $(\mathrm{N}=5)$. The (a) indicates a $\mathrm{p}$ value $<0.05$ compared to the control group.

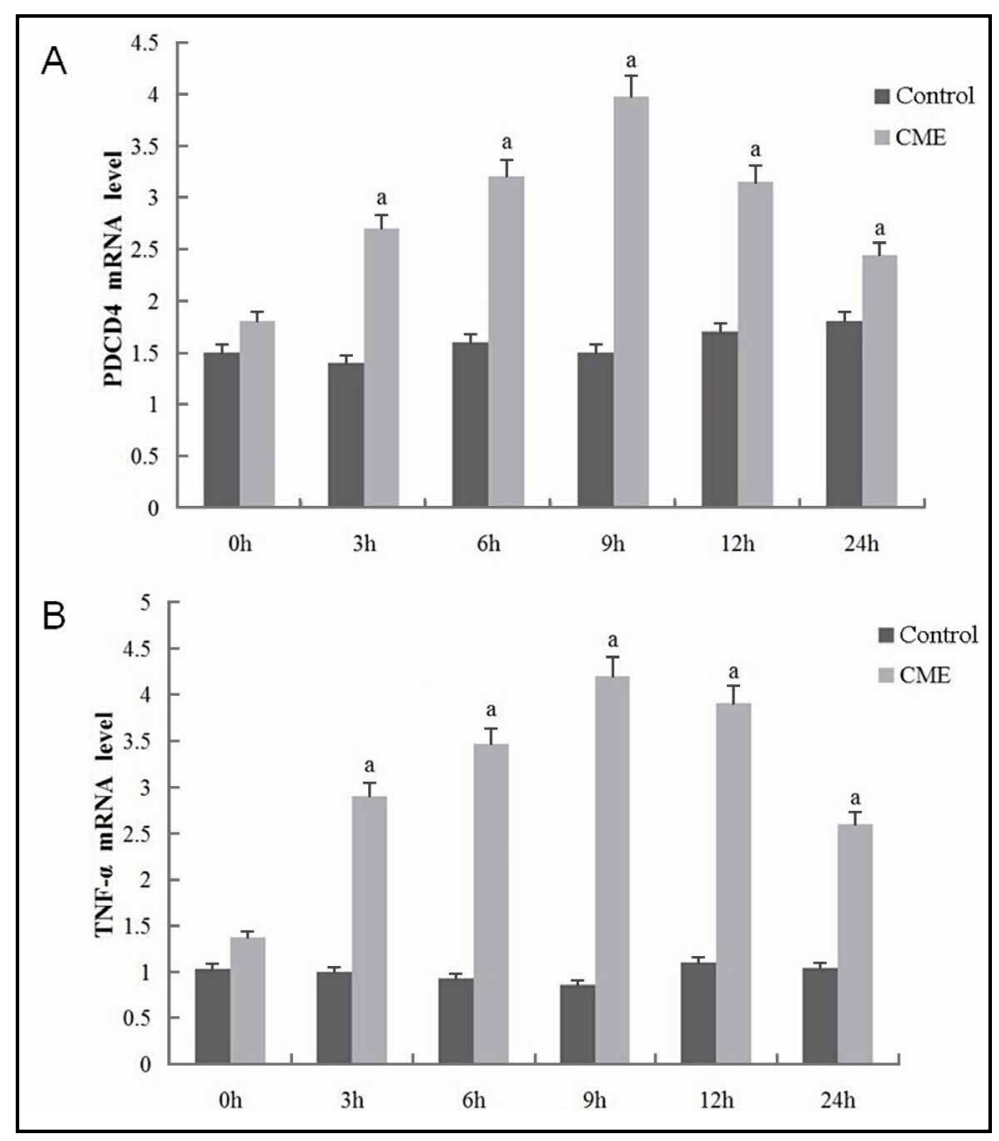

Fig. 4. PDCD4 and TNF- $\alpha$ mRNA transcription in myocardial tissues as detected by qPCR in the CME plus PDCD4siRNA and CME plus controlsiRNA groups. (A) PDCD4 (B) TNF- $\alpha$ normalized against GAPDH. Data are mean \pm SEM $(\mathrm{N}=5)$. The (a) indicates a $\mathrm{p}$ value $<0.05$ compared to the control-siRNA group.
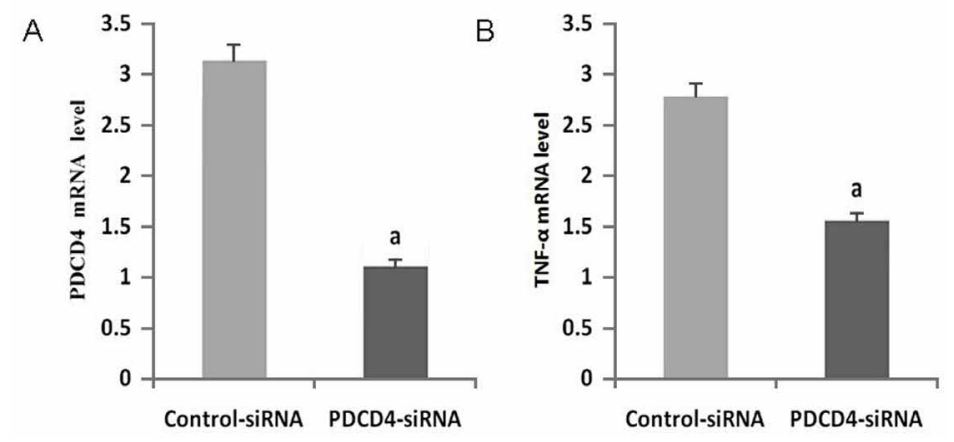

significantly attenuated the enhanced myocardial PDCD4 and TNF- $\alpha$ mRNA expression $9 \mathrm{~h}$ after CME modeling (Fig. 4, $\mathrm{P}<0.05$ ).

The myocardial expression of PDCD4, TNF- $\alpha$ and caspase-3 proteins

As determined by western blot analysis, the myocardial levels of PDCD4, TNF- $\alpha$ and caspase-3 proteins were significantly increased in the minipigs of the CME group compared with those of the control group (Fig. 5, $\mathrm{P}<0.05$ ). Compared with $0 \mathrm{~h}$, the myocardial PDCD4 and TNF- $\alpha$ proteins increased at $3 \mathrm{~h}$, peaked at $9 \mathrm{~h}$, and were still increased at 12 and $24 \mathrm{~h}$ after the procedure $(\mathrm{P}<0.05)$. Moreover, the expression of activated caspase-3 LAO increased at $3 \mathrm{~h} 6 \mathrm{~h} 9 \mathrm{~h} 12 \mathrm{~h}$, and $24 \mathrm{~h}$ in the CME pigs compared with the controls and reached the highest level at $12 \mathrm{~h}$ after CME. Interestingly, PDCD4-siRNA treatment significantly attenuated the enhanced PDCD 4 and TNF- $\alpha$ protein expression $9 \mathrm{~h}$ after CME modeling (Fig. 6, $\mathrm{P}<0.05$ ). 
Fig. 5. Dynamic changes of PDCD4, TNF- $\alpha$ and caspase-3 protein expression in the myocardium of the CME and control groups as detected by western blotting. (A) PDCD4, (B) TNF- $\alpha$ and (C) caspase-3 normalized against GAPDH. Data are mean \pm SEM $(\mathrm{N}=5)$. The (a) indicates a $\mathrm{p}$ value $<0.05$ compared to the control group.

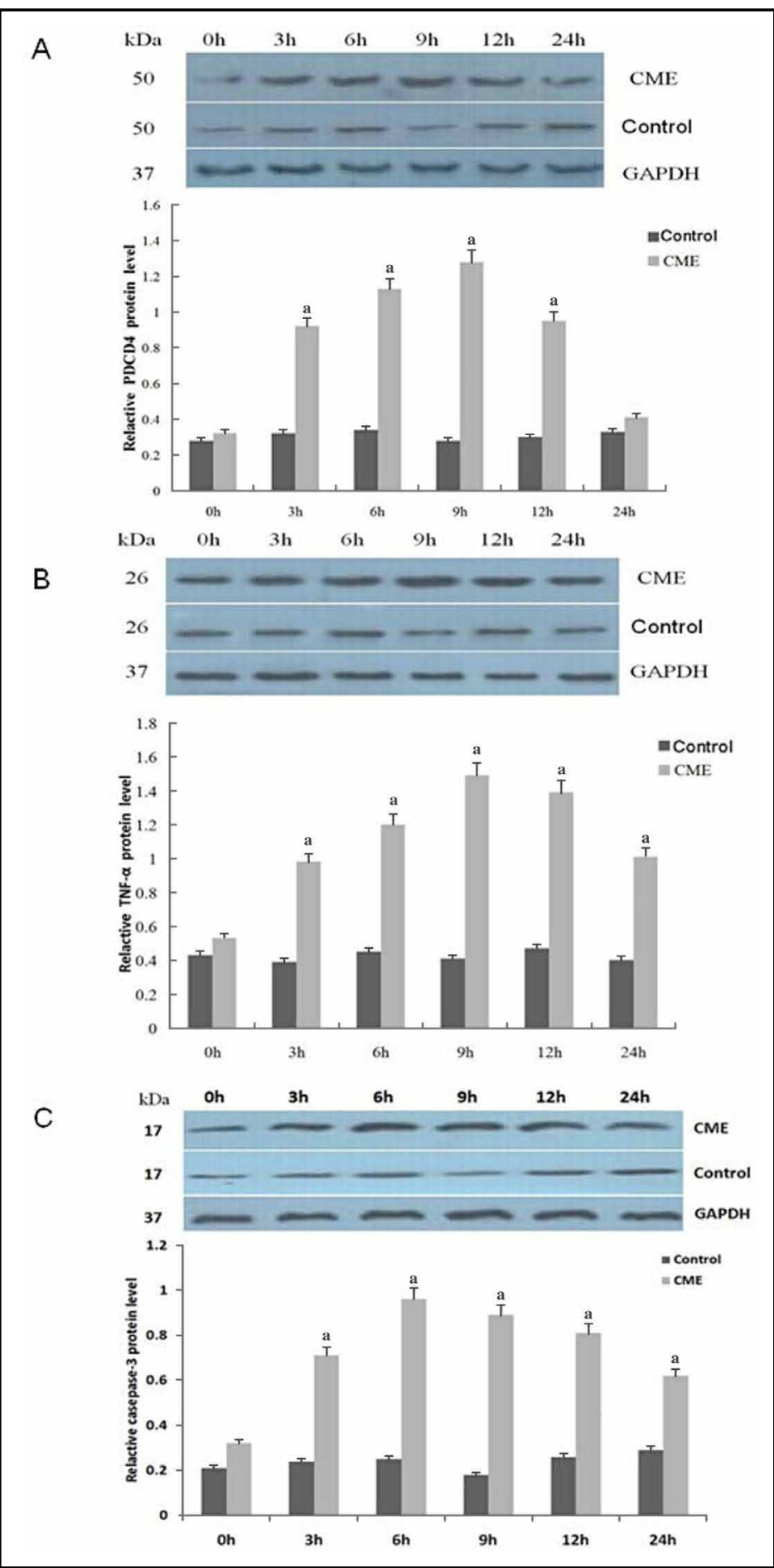

However, there was no significant difference in the expression of activated caspase- 3 in the minipigs from the CME plus PDCD4-siRNA group compared with those from the CME plus control-siRNA group.

Linear correlation analysis

Linear correlation analysis indicated that the PDCD4 was negatively correlated with LVEF $(r=-0.618, P=0.018)$, while TNF- $\alpha$ was negatively correlated with LVEF $(r=-0.692, P=$ $0.013)$. TNF- $\alpha$ was positively correlated with PDCD4 ( $r=0.720, P=0.009)$. 


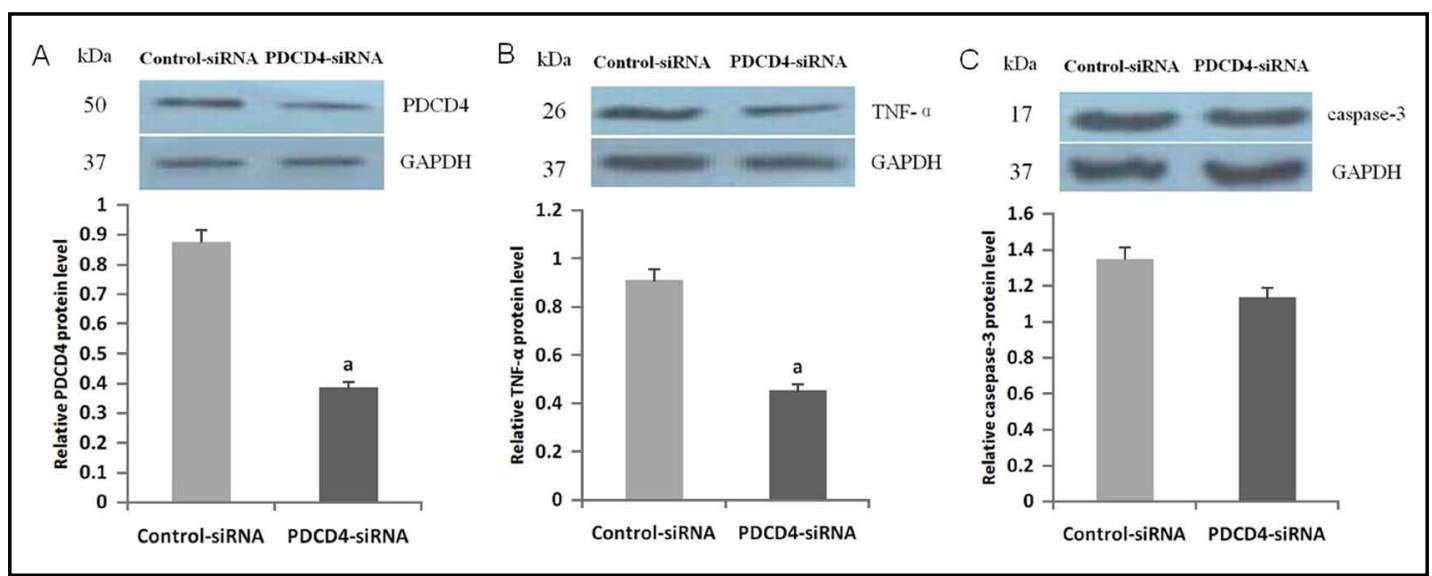

Fig. 6. PDCD4, TNF- $\alpha$ and caspase-3 protein expression in the myocardium of the CME plus PDCD4-siRNA and CME plus control-siRNA groups as detected by western blotting. (A) PDCD4, (B) TNF- $\alpha$ and (C) caspase- 3 normalized against GAPDH. Data are mean \pm SEM $(\mathrm{N}=5)$. The $(\mathrm{a})$ indicates a $\mathrm{p}$ value $<0.05$ compared to the control-siRNA group.

\section{Discussion}

To our knowledge, this is the first report on the involvement of PDCD4 in the pathogenesis of CME. We provide the first evidence that there is significant up-regulation and dynamic changes of PDCD4 in the pathogenesis of CME-related cardiac dysfunction in minipigs. Consistent with previous reports, we also found that the expression of TNF- $\alpha$ and caspase- 3 was stimulated in the myocardial tissues of CME pigs.

CME results in perfusion-contraction mismatch and is associated with an inflammatory response [8]. The embolization of debris into the coronary microcirculation is accepted as the initial mechanism for cardiac dysfunction during the acute phase of CME, while the inflammatory activation is responsible for the regional contractile dysfunction after CME. Increased myocardial TNF- $\alpha$ has been identified as a potential contributor to contractile dysfunction and subsequent ventricular remodeling. The intracoronary infusion of exogenous TNF- $\alpha$ in the absence of microembolization induces similar progressive myocardial dysfunction; moreover, pretreatment with TNF- $\alpha$ antibodies prevents myocardial dysfunction following CME $[10,16]$. In our previous studies, we found that p38 MAPK is significantly activated by CME and that inhibition of p38 MAPK partly depresses the TNF- $\alpha$ expression, preserving cardiac contractile function [17]. Lu et al. also reported that higher levels of TNF- $\alpha$ and IL-1 $\beta$ in rats following CME are associated with the development of myocardial contractile dysfunction [18]. Consistent with previous findings, we also found that TNF- $\alpha$ was upregulated in the CME group in this study, paralleling the impairment of myocardial contractile function. Interestingly, we did not observe any difference in myocardial infarction area between the CME and control groups, implying that the ischemic injury and subsequent myocardial infarction were unlikely to be a major contributor to the cardiac dysfunction after the induction of CME.

PDCD4 is an important mediator of cancer cell apoptosis at early stages [19, 20]. PDCD4 is one of the target genes of miR-21, which is related to the miR-21-mediated anti-apoptotic effect on vascular smooth muscle cells [21]. The roles of PDCD4 as a target gene of miR-21 and mediator in miR-21-mediated cardiac cell protection have also been confirmed in both in vitro and in vivo cardiac I/R models. The protective effect of miR-21 against ischemiainduced cardiomyocyte damage was confirmed in vivo by attenuated cell apoptosis in the border and infarcted areas in a rat model of myocardial infarction [12]. Recently, we 
found that intensive atorvastatin treatment reduced the post-PCI myocardial inflammatory response in patients with unstable angina, possibly by inhibiting PDCD4 expression in CD4 ${ }^{+}$ T lymphocytes [13]. In this study, we also found that the expression of TNF- $\alpha$ was positively correlated with the expression of PDCD4 after CME. In this study, down-regulation of PDCD 4 by PDCD4-siRNA treatment suppressed the expression of myocardial PDCD4 and TNF- $\alpha$ after $\mathrm{CME}$, which further supports the conclusion of the atorvastatin study. Based on the above evidence, it is plausible that PDCD4 impairs LV dysfunction after CME by inducing the expression of TNF- $\alpha$. Furthermore, we found that there was no significant change in the level of activated caspase- 3 after down-regulation of PDCD4, indicating that regulation of myocardial apoptosis may not be a possible mechanism underlying the therapeutic role of PDCD4-siRNA during CME.

There are two main limitations of the present study. First, the polymer microspheres we used to induce the CME model did not accurately mimic the natural process of in vivo microembolization in patients, which also includes complex interactions of platelet activation, endothelial dysfunction, and plaque debris with cholesterol crystals. Second, we only measured PDCD4 expression in the myocardial tissues, and no causative relationships between PDCD4 and CME could be illustrated. Further work is needed to unveil the mechanism responsible for this phenomenon.

In summary, our study suggests that PDCD4 is significantly up-regulated during the pathogenesis of cardiac dysfunction after CME in minipigs, which may be a key mechanism of CME-induced cardiac dysfunction and is mediated at least partially by the induction of TNF- $\alpha$.

\section{Acknowledgments}

This study was supported by a grant from the National Natural Science Foundation of China (Grant no. 81260042) and Youth Science Foundation of Guangxi Medical University (Grant no. GXMUYSF201321).

\section{Disclosure Statement}

This manuscript has not been published elsewhere in whole or in part. All authors have read and approved the content and agree to submit the manuscript for consideration for publication in this journal. The authors have no conflicts of interest to declare.

\section{References}

$\checkmark 1$ Heusch G, Kleinbongard P, Böse D, Levkau B, Haude M, Schulz R, Erbel R: Coronary microembolization: from bedside to bench and back to bedside. Circulation 2009;120:1822-1836.

-2 Böse D, von Birgelen C, Zhou XY, Schmermund A, Philipp S, Sack S, Konorza T, Möhlenkamp S, Leineweber K, Kleinbongard P, Wijns W, Heusch G, Erbel R: Impact of atherosclerotic plaque composition on coronary microembolization during percutaneous coronary interventions. Basic Res Cardiol 2008;103:587-597.

-3 Ma JY, Qian JY, Jin H, et al: Acute hyperenhancement on delayed contrast-enhanced magnetic resonance imaging is the characteristic sign after coronary microembolization. Chin. Med J [Engl] 2009;122:687-691.

-4 Otto S, Seeber M, Fujita B, Kretzschmar D, Ferrari M, Goebel B, Figulla HR, Poerner TC: Microembolization and myonecrosis during elective percutaneous coronary interventions in diabetic patients: an intracoronary Doppler ultrasound study with 2-year clinical follow-up. Basic Res Cardiol 2012;107:289.

-5 Bahrmann P, Werner GS, Heusch G, Ferrari M, Poerner TC, Voss A, Figulla HR: Detection of coronary microembolization by Doppler ultrasound in patients with stable angina pectoris undergoing elective percutaneous coronary interventions. Circulation 2007;115:600-608. 


\begin{tabular}{|c|c|c|}
\hline Cellular Physiology & Cell Physiol Biochem 2014;34:533-542 & \\
\hline and Biochemistry & $\begin{array}{l}\text { DOI: 10.1159/000363020 } \\
\text { Publisnea onine: August 08, } 2014\end{array}$ & $\begin{array}{l}\text { O } 2014 \text { S. Karger AG, Basel } \\
\text { www.karger.com/cpb }\end{array}$ \\
\hline
\end{tabular}

6 Li L, Zhao X, Lu Y, Huang W, Wen W: Altered expression of pro- and anti-inflammatory cytokines is associated with reduced cardiac function in rats following coronary microembolization. Mol Cell Biochem 2010;342:183-190.

7 Dörge H, Schulz R, Belosjorow S, Post H, van de Sand A, Konietzka I, Frede S, Hartung T, Vinten-Johansen J, Youker KA, Entman ML, Erbel R, Heusch G. Coronary microembolization: the role of TNF-alpha in contractile dysfunction. J Mol Cell Cardiol. 2002;34:51-62.

8 Dörge H, Neumann T, Behrends M, Skyschally A, Schulz R, Kasper C, Erbel R, Heusch G: Perfusioncontraction mismatch with coronary microvascular obstruction: role of inflammation. Am J Physiol Heart Circ Physiol 2000;279:H2587-H2592.

-9 Canton M, Skyschally A, Menabò R, Boengler K, Gres P, Schulz R, Haude M, Erbel R, Di Lisa F, Heusch G: Oxidative modification of tropomyosin and myocardial dysfunction following coronary microembolization. Eur Heart J 2006;27:875-881.

-10 Thielmann M, Dörge H, Martin C, Belosjorow S, Schwanke U, van De Sand A, Konietzka I, Büchert A, Krüger A, Schulz R, Heusch G: Myocardial dysfunction with coronary microembolization: signal transduction through a sequence of nitric oxide, tumor necrosis factor-alpha, and sphingosine. Circ Res 2002;90:807813.

11 Wang Q, Dong Z, Liu X, Song X, Song Q Shang Q Jiang Y, Guo C, Zhang L: Programmed cell death-4 deficiency prevents diet-induced obesity, adipose tissue inflammation, and insulin resistance. Diabetes 2013;62:4132-4143.

12 Cheng Y, Zhu P, Yang J, Liu X, Dong S, Wang X, Chun B, Zhuang J, Zhang C: Ischaemic preconditioningregulated miR-21 protects heart against ischaemia/reperfusion injury via anti-apoptosis through its target PDCD4. Cardiovasc Res 2010;87:431-439.

13 Su Q Li L, Liu Y, Zhou Y, Wang J, Sun Y. Effect of intensive atorvastatin therapy on periprocedural PDCD4 expression in CD4+ T lymphocytes of patients with unstable angina undergoing percutaneous coronary intervention. Cardiology 2014;127:169-75.

-14 Herrmann J, Lerman A, Baumgart D, Volbracht L, Schulz R, von Birgelen C, Haude M, Heusch G, Erbel R: Preprocedural statin medication reduces the extent of periprocedural non-Q-wave myocardial infarction. Circulation 2002;106:2180-2183.

15 Chen C, Hu Q, Yan J, Yang X, Shi X, Lei J, Chen L, Huang H, Han J, Zhang JH, Zhou C: Early inhibition of HIF-1alpha with small interfering RNA reduces ischemic-reperfused brain injury in rats. Neurobiol Dis 2009;33:509-517.

16 Skyschally A, Gres P, Hoffmann S, Haude M, Erbel R, Schulz R, Heusch G: Bidirectional role of tumor necrosis factor-alpha in coronary microembolization: progressive contractile dysfunction versus delayed protection against infarction. Circ Res 2007;100:140-146.

17 Li L, Qu N, Li DH, et al: Coronary microembolization induced myocardial contractile dysfunction and tumor necrosis factor-alpha mRNA expression partly inhibited by SB203580 through a p38 mitogen-activated protein kinase pathway. Chin. Med J [Engl] 2011;124:100-105.

-18 Lu Y, Li L, Zhao X, Huang W, Wen W: Beta blocker metoprolol protects against contractile dysfunction in rats after coronary microembolization by regulating expression of myocardial inflammatory cytokines. Life Sci 2011;88:1009-1015.

19 Zhang Z, Zha Y, Hu W, Huang Z, Gao Z, Zang Y, Chen J, Dong L, Zhang J: The autoregulatory feedback loop of MicroRNA-21/Programmed cell death Protein 4/activation Protein-1 (MiR-21/PDCD4/AP-1) as a driving Force for hepatic fibrosis development. J Biol Chem 2013;288:37082-37093.

20 Hilliard A, Hilliard B, Zheng SJ, Sun H, Miwa T, Song W, Göke R, Chen YH: Translational regulation of autoimmune inflammation and lymphoma genesis by programmed cell death 4 . J Immunol 2006;177:8095-8102.

21 Dong S, Cheng Y, Yang J, Li J, Liu X, Wang X, Wang D, Krall TJ, Delphin ES, Zhang C: MicroRNA expression signature and the role of microRNA-21 in the early phase of acute myocardial infarction. J Biol Chem 2009;284:29514-29525. 